\title{
Restoration of the Severely Decayed Tooth Using Crown Lengthening with Simultaneous Tooth-Preparation
}

\author{
Jun-Beom Park ${ }^{\mathrm{a}}$
}

\section{ABSTRACT}

This clinical case describes a treatment approach that combines, in a single appointment, a crown lengthening procedure and the final crown preparation for the restoration of a severely decayed tooth. This approach allows for a more accurate placement of the crown margin in relation to the bone crest while reducing treatment time. (Eur J Dent 2010;4:197-201)

Key words: Periodontal surgery; Crown; Margin.

\section{INTRODUCTION}

Periodontal surgical preparation may be required for specific problems in restorative dentistry such as subgingival caries, crown fracture, and inadequate crown length. ${ }^{1}$ Crown lengthening is often necessary to provide adequate room for crown preparation and reestablishment of the biologic width between the crown margin and the bone crest. ${ }^{2}$ Traditionally, these procedures are carried out in a staged manner with the crown lengthening procedure done before finalization of the tooth preparation. When this approach is used,

a Department of Pharmaceutical Sciences, College of Pharmacy, University of Michigan, Ann Arbor, MI, USA.

- Corresponding author: Jun-Beom Park DDS, MSD, PhD Visiting Scientist at Department of Pharmaceutical Sciences, College of Pharmacy, University of Michigan. 1664 Mclntyre Drive, Ann Arbor, MI, 48105, USA.

Phone: +1-734-565-9775

E-mail: jbassoonisdyahoo.co.kr the final location of the crown has not been established, thereby making it difficult to evaluate the exact amount of crown lengthening necessary to complete the restorative phase successfully and maintain periodontal health thereafter. ${ }^{3}$

In this case, tooth preparation and crown lengthening procedure were performed at the same appointment to allow for more optimal results and to reduce chair time.

\section{CASE REPORT}

A 21-year-old male patient presented with a complaint of loss of crown structure. The patient had a long history of dental caries; his medical history was noncontributory.

Upon clinical examination of the right mandibular region, it was noted that the pulp of the first molar had been exposed by decay, and a pulp polyp had formed (Figure 1). The tooth was un- 
responsive to sensitivity testing with cold or with an electric pulp tester (Parkell, Farmingdale, NY, USA). Plaque could be seen, and isolated bleeding spots were noticed when a North Carolina periodontal probe (Hu-Friedy, Chicago, IL, USA) was passed along the gingival margin. The keratinized tissue on the buccal side was more than $3 \mathrm{~mm}$ wide, and clinical probing depths ranged from 3 to $4 \mathrm{~mm}$. Radiographic examination demonstrated an apparent radiolucency in the apical third of the tooth (Figure 2). A diagnosis of chronic hyperplastic pulpitis was made.

A treatment plan was developed that included endodontic treatment, core build-up, crown lengthening procedure, and fabrication of a single crown for the restoration of the tooth. The patient was given a detailed explanation concerning the suggested treatment procedure and prognosis, and consent for treatment was received.

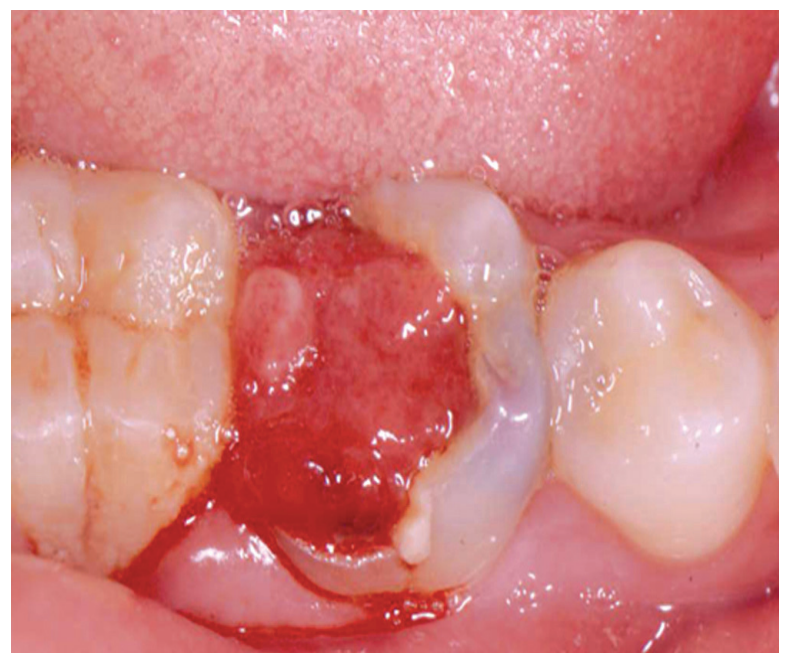

Figure 1. Clinical photograph taken at the initial visit showing severe dental caries with granulation tissue.

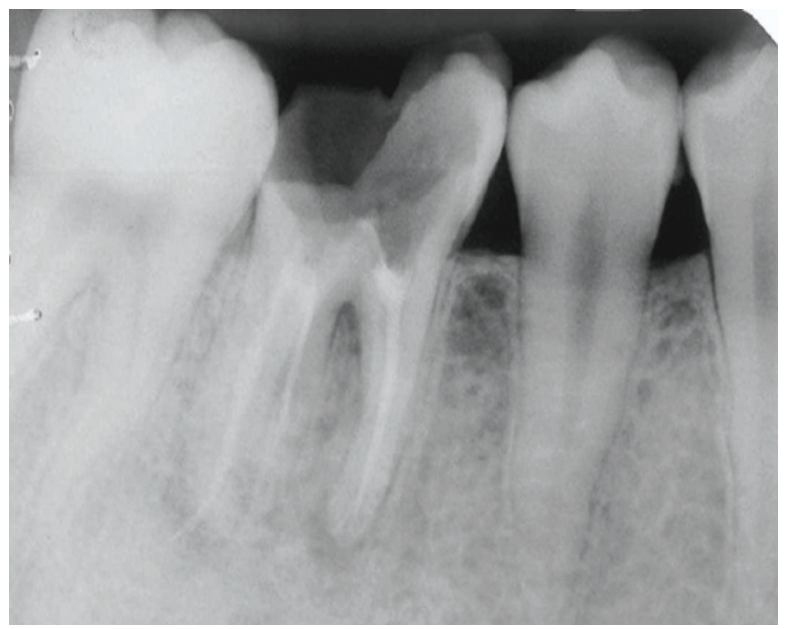

Figure 3. Radiograph of the lower right first molar filled with gutta-percha points and sealer using a lateral condensation technique.

\section{Surgical procedure}

After removing the polyp, a conventional access cavity was prepared in the occlusal surface of the first molar with a 330-carbide bur and widened with an Endo-Z bur (Dentsply Maillefer, Tulsa, OK, USA) to enhance visibility of the root canal system. Irrigation of the canal was done several times with $5 \%$ sodium hypochlorite, and the last irrigation solution was left in the canal to dissolve organic material. Determination of the working length was done using an electronic apex locator (Root ZX®, J Morita Corporation, Kyoto, Japan) and the radiograph. Canal enlargement was performed using a hand file, and the root canals were filled with gutta-percha points (Diadent, Seoul, Korea) and sealer (AH26, Dentsply, Konstanz, Germany) using a lateral condensation technique (Figure 3). A post (ParaPost, Coltène/Whaledent Inc., Cuyahoga Falls, OH, USA) was inserted in the mesio-buccal

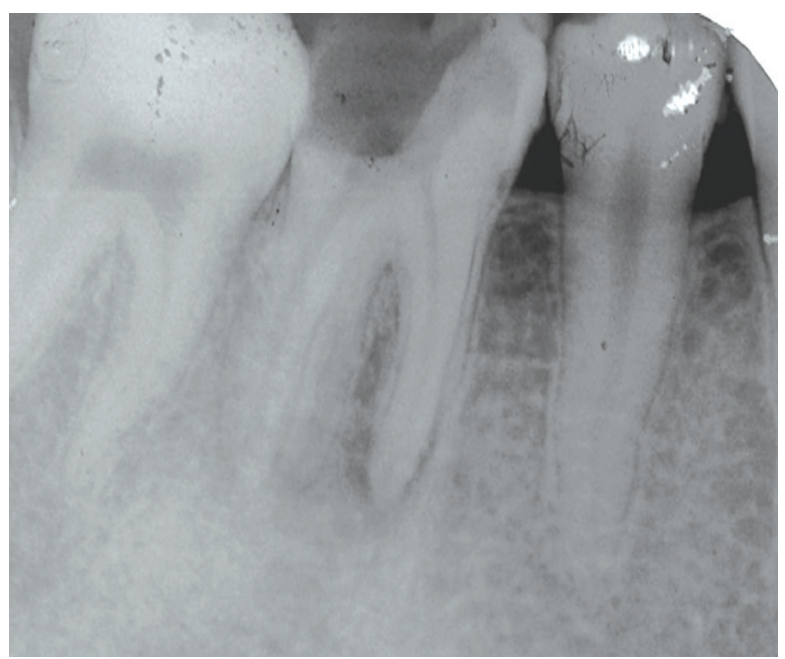

Figure 2. Preoperative radiograph of the lower right first molar.

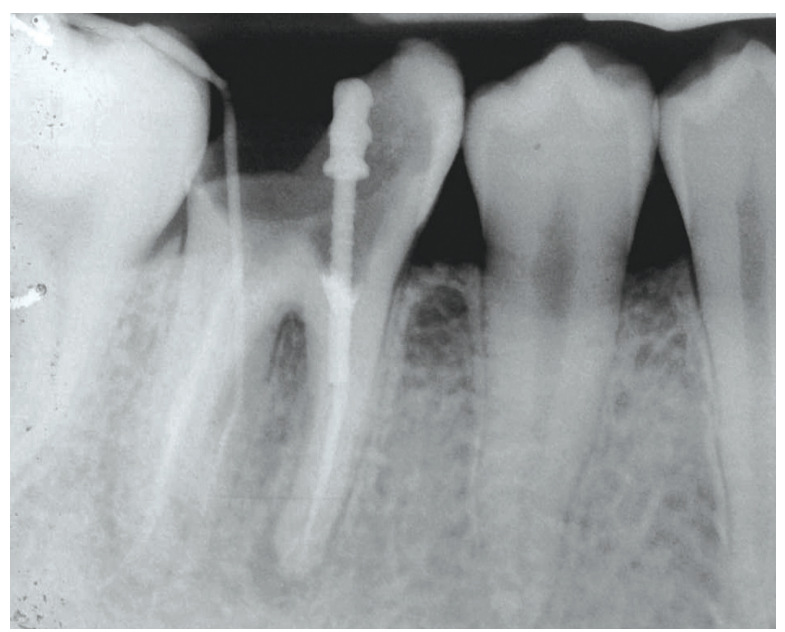

Figure 4. Radiograph with the post in place. 
canal (Figure 4), and the core build-up was done with a light-cured resin (Fuji II LC, GC, Alsip, IL, USA) added in layers (Figure 5).

Following an injection of $2 \%$ lidocaine with 1:100,000 epinephrine local anesthetic, a fullthickness flap was reflected. Crown preparation was done and ostectomy was performed to create an appropriate biologic width (Figure 6). Sutures were placed, and routine postoperative instructions were given (Figure 7). The patient was prescribed amoxicillin $500 \mathrm{mg} 3$ times per day for 5 days, mefenamic acid $500 \mathrm{mg}$ initially, then mefenamic acid $250 \mathrm{mg} 4$ times per day for 5 days, and $0.12 \%$ chlorhexidine digluconate 3 times per day for 2 weeks.

\section{Clinical observations}

Two weeks after surgery, any remaining sutures were removed. The surgical site showed

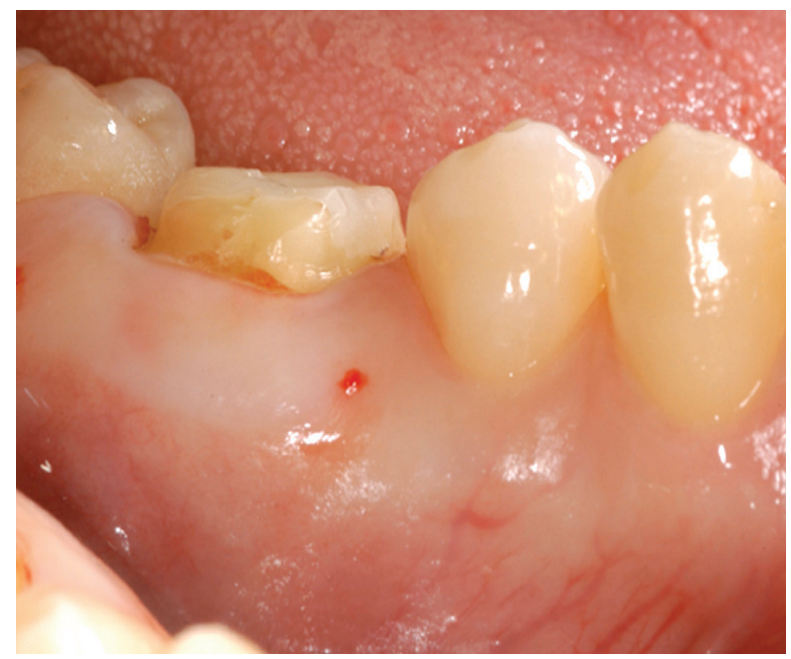

Figure 5. Buccal view with a resin core.

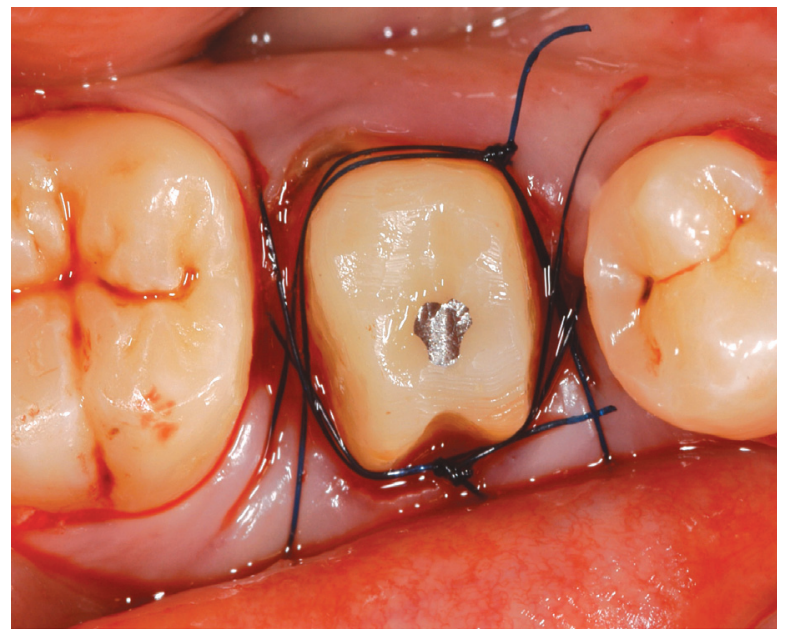

Figure 7. Occlusal view of sutured surgical site showing the prepared tooth. good healing (Figure 8). A temporary prosthesis was fabricated and cemented (Temp-Bond, Kerr Corp., Romulus MI, USA). A two-month postoperative occlusal view showed good soft tissue healing (Figure 9).

The final evaluation at three months shows a healthy state of soft tissue with good adaptation of the final restoration (Figure 10).

\section{DISCUSSION}

Crown lengthening is performed to achieve adequate room for crown preparation and reestablishment of the biologic width. ${ }^{2}$ Traditional staged approach forces the periodontist to estimate the approximate location of the crown margin. This case report chronicles the simultaneous performance of the crown lengthening procedure and tooth preparation to allow a more accurate placement of the crown margin in relation to the bone

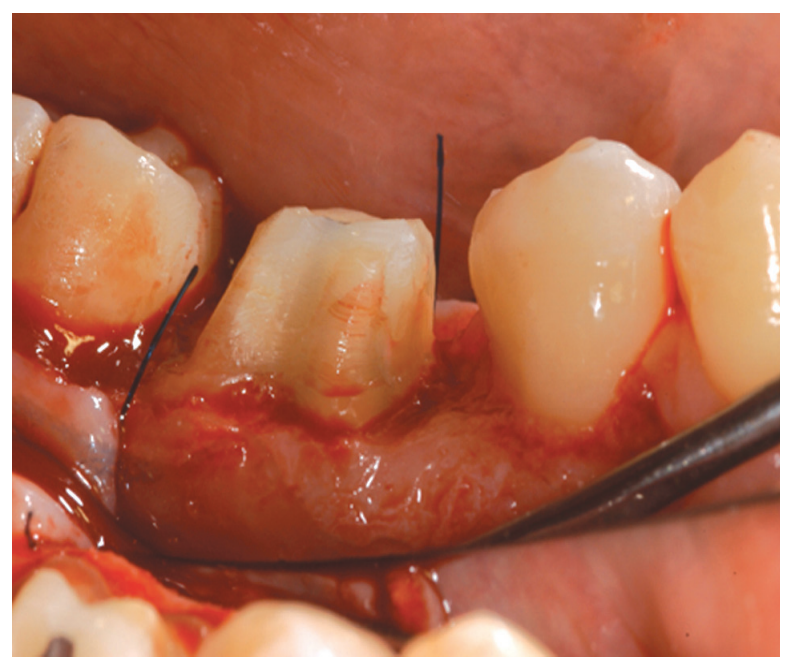

Figure 6. Crown preparation and crown lengthening procedure were done after a full-thickness flap was reflected.

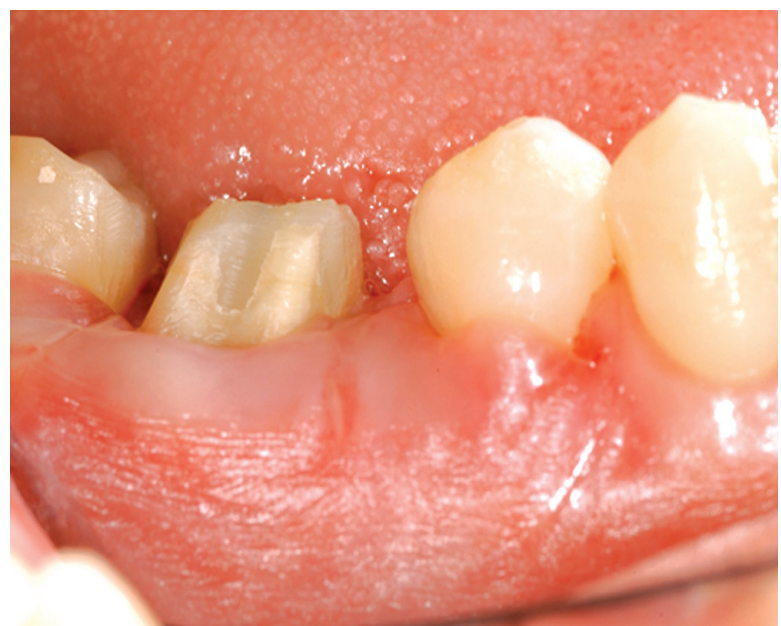

Figure 8. A fourteen-day postoperative buccal view showing good healing state. 
crest, thus enhancing the contours of the final restoration. $^{3}$

This approach requires that treatment planning be done in a multidisciplinary fashion, taking into account the amount of bone removal required and its effect, not only on the tooth to be restored but also on adjoining teeth. If radiographs show that excessive osteoctomy is required and would not allow for maintenance of an acceptable bony architecture, then extraction should be recommended even if the patient wishes to keep the tooth in function by any means. ${ }^{1}$ Consideration of the root trunk of a second mandibular molar is especially important since it is generally longer than that of the first molar. ${ }^{4}$

Another goal of treatment should be the creation of a band of keratinized tissue wide enough to provide increased resistance to the periodontium and contribute to the stabilization of the gingival margin position. ${ }^{5}$ Improvement of oral hygiene performance can be achieved with the increase

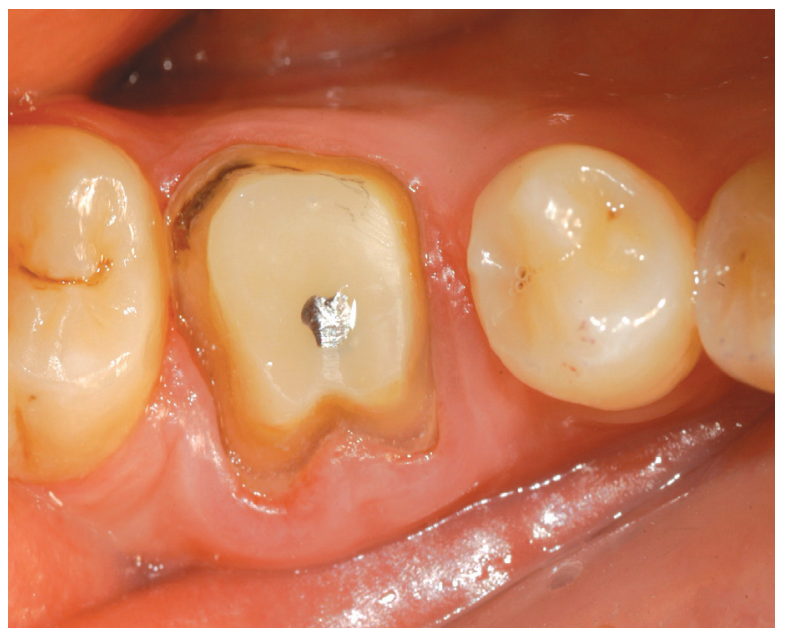

Figure 9. A two-month postoperative occlusal view showing good healing.

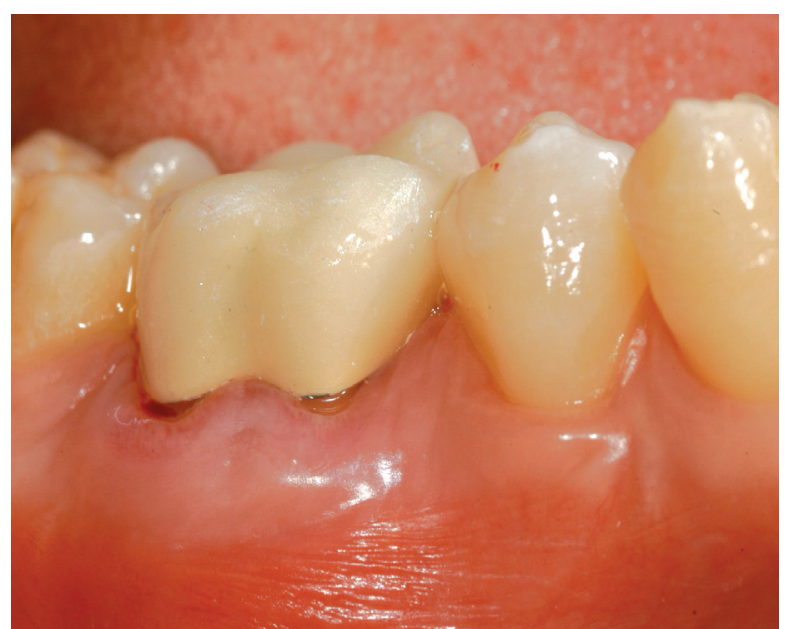

Figure 10. Buccal view with the permanent restoration at the final evaluation. of keratinized tissue. ${ }^{6}$ In this case, the available width of keratinized tissue was more than $3 \mathrm{~mm}$, and no additional procedures were required.

Subgingival margins are associated with unfavorable periodontal reaction, even among patients receiving regular preventive dental care. ${ }^{7}$ Supragingival margin placement was suggested for all restorative margins to avoid iatrogenic periodontal disease. ${ }^{8}$ Plaque from the subgingival margin group had significantly higher counts of spirochetes, fusiforms, rods, and filaments as compared to gingival or supragingival margins. ${ }^{9}$ Supragingival margin was used in this case because a supragingival location of the crown margin showed lower signs of inflammation. ${ }^{10}$

\section{CONCLUSIONS}

More accurate determination of the margin with the reduction of treatment time was achieved by applying tooth preparation at the time of crown lengthening procedure. This approach may be considered an option when a multidisciplinary approach is possible. Large case-control studies over an extended period of time are necessary to establish whether this procedure offers long-term benefits to patients.

\section{REFERENCES}

1. Kaldahl WB, Becker CM, Wentz FM. Periodontal surgical preparation for specific problems in restorative dentistry. $J$ Prosthet Dent 1984;51:36-41.

2. Ross SE, Garguilo A. The surgical management of the restorative alveolar interface. Int $J$ Periodontics Restorative Dent 1982;2:8-31.

3. Mammen S, Meyer R. Single-appointment crown lengthening and restorative procedure under the rubber dam. Gen Dent 2001;49:215-220.

4. Dibart S, Capri D, Kachouh I, Van Dyke T, Nunn ME. Crown lengthening in mandibular molars: A 5-year retrospective radiographic analysis. J Periodontol 2003;74:815-821.

5. Hassell TM. Tissues and cells of the periodontium. Periodontol 2000 1993;3:9-38.

6. Wei PC, Laurell L, Geivelis M, Lingen MW, Maddalozzo D. Acellular dermal matrix allografts to achieve increased attached gingiva. Part 1. A clinical study. J Periodontol 2000;71:1297-1305.

7. Bader JD, Rozier RG, McFall WT, Jr., Ramsey DL. Effect of crown margins on periodontal conditions in regularly attending patients. J Prosthet Dent 1991;65:75-79.

8. Reeves WG. Restorative margin placement and periodontal health. J Prosthet Dent 1991;66:733-736. 
9. Ericson $G$, Nilson H, Bergman B. Cross-sectional study of patients fitted with fixed partial dentures with special reference to the caries situation. Scand J Dent Res 1990;98:8-16.

10. Muller HP. The effect of artificial crown margins at the gingival margin on the periodontal conditions in a group of periodontally supervised patients treated with fixed bridges. J Clin Periodontol 1986;13:97-102. 